\title{
Cortical responses elicited by luminance and compound stimuli modulated by pseudo-random sequences: comparison between normal trichromats and congenital red-green color blinds
}

\author{
Bárbara B. O. Risuenho ${ }^{1}$, Letícia Miquilini ${ }^{1}$, Eliza Maria C. B. Lacerda ${ }^{1}$, Luiz Carlos L. Silveira ${ }^{1,2,3}$ and \\ Givago S. Souza ${ }^{1,2}$ *
}

1 Instituto de Ciências Biológicas - Universidade Federal do Pará, Belém, Brazil

${ }^{2}$ Núcleo de Medicina Tropical - Universidade Federal do Pará, Belém, Brazil

${ }^{3}$ Universidade Ceuma, São Luís, Brazil

\section{Edited by:}

Marcelo Fernandes Costa

Universidade de São Paulo, Brazil

Reviewed by:

Sophie Wuerger, University of

Liverpool, UK

Thomas Wachtler,

Ludwig-Maximilians-Universität

München, Germany

\section{${ }^{*}$ Correspondence:}

Givago S. Souza, Núcleo de Medicina

Tropical - Universidade Federal do

Pará, Av. Generalíssimo Deodoro 92

Umarizal 66055-240, Belém, Pará,

Brazil

e-mail: givagosouza@ufpa.br
Conventional pattern-reversal visual evoked cortical potential (VECP) shows positivity for luminance and chromatic equiluminant stimuli while conventional pattern-onset VECP shows positivity for luminance pattern-onset and negativity for chromatic pattern-onset. We evaluated how the presentation mode affects VECPs elicited by luminance and compound (luminance plus chromatic) pseudo-random stimulation. Eleven normal trichromats and 17 red-green color-blinds were studied. Pattern-reversal and pattern-onset luminance and compound (luminance plus red-green) gratings were temporally modulated by $\mathrm{m}$ sequence. We used a cross-correlation routine to extract the first order kernel (K1) and the first and second slices of the second order kernel (K2.1 and K2.2, respectively) from the $\mathrm{VECP}$ response. We integrated the amplitude of $\mathrm{VECP}$ components as a function of time in order to estimate its magnitude for each stimulus condition. We also used a normalized cross-correlation method in order to test the similarity of the VECP components. The VECP components varied with the presentation mode and the presence of red-green contrast in the stimuli. In trichromats, for compound conditions, pattern-onset K1, K2.1, and K2.2, and pattern-reversal K2.1 and K2.2 had negative-dominated waveforms at $100 \mathrm{~ms}$. Small negativity or small positivity were observed in dichromats. Trichromats had larger VECP magnitude than color-blinds for compound pattern-onset K1 (with large variability across subjects), compound pattern-onset and pattern-reversal K2.1, and compound patternreversal K2.2. Trichromats and color-blinds had similar VECP amplitude for compound pattern-reversal $\mathrm{K} 1$ and compound pattern-onset K2.2, as well as for all luminance conditions. The cross-correlation analysis showed high similarity between waveforms of compound pattern-onset K2.1 and pattern-reversal K2.2 as well as pattern-reversal K2.1 and K2.2. We suggest that compound pattern-reversal K2.1 is an appropriate response to study red-green color-opponent activity.

Keywords: evoked potential, pseudo-random VECP, pattern-onset VECP, pattern-reversal VECP, color vision, trichromacy, daltonism

\section{INTRODUCTION}

Using pseudo-random stimulation it was possible to elicit negative-dominated visual evoked cortical potentials (VECP) for chromatic equiluminant contrast using both pattern-onset and pattern-reversal stimuli (Gerth etal., 2003). These findings differed from those obtained with conventional periodical stimulation (Carden et al., 1985; Suttle and Harding, 1999). When conventional periodical stimuli are used, such as sinusoidal gratings, luminance, and chromatic equiluminant pattern-reversal as well as luminance pattern-onset stimulation elicited positivedominated VECP at around $100 \mathrm{~ms}$ while chromatic equiluminant pattern-onset stimulation elicited negative-dominated VECP at the same latency (Carden et al., 1985; Kulikowski and Parry, 1987; Murray et al., 1987; Kulikowski et al., 1989). Since the chromatic equiluminant pattern-onset VECP usually shows higher signal-tonoise ratio compared to chromatic equiluminant pattern-reversal VECP and also exhibit inverse polarity compared to luminance pattern-onset VECP, many have used this stimulation mode to study the mechanism of chromatic equiluminant transient VECPs (Carden et al., 1985; Kulikowski and Parry, 1987; Murray et al., 1987; Berninger et al., 1989; Kulikowski et al., 1989, 1996; Rabin et al., 1994; Porciatti and Sartucci, 1999; Gomes et al., 2006, 2008, 2010; Souza et al., 2008).

McKeefry et al. (1996) discussed about the activation of chromatic and achromatic mechanisms by pattern reversal and pattern onset-offset stimulations. They based their suggestions in the features of response from tonic and phasic cells in the visual system (Gouras, 1968; Dreher et al., 1976; Kaplan and Shapley, 
1982, 1986; Hicks et al., 1983). They consider that for some stimulus selective condition the dichotomy between tonic/sustained cells and phasic/transient cells overlap with magnocellular (luminance) and parvocellular (chromatic) activity, respectively. Tonic cells has larger responses for the onset of the stimulus than for its offset due the sustained response is longer as long as the stimulus is on, and it has low responsivity to contrast reversal modulated by temporal square-wave function due its sustained nature of their response. Phasic cells had similar responses for the stimulus onset and offset due their transient response, but they has higher responses for contrast reversal with square-wave temporal modulation. McKeefry et al. (1996) found that the chromatic onset-offset VECP is dominated by a fundamental component, while the achromatic onset-offset VECP had a second harmonic additionally to the fundamental component. For pattern reversal VECPs, both chromatic and achromatic responses, had high second harmonic, which peaked at the achromatic stimulus condition and was minimum at the chromatic isoluminance.

Until now, only homogenous fields or complex patterns were used to compose pseudo-random stimuli to investigate chromatic cortical responses in different visual field sectors (Baseler and Sutter, 1997; Gerth et al., 2003). For spatial vision, additionally to dartboard stimulus (Baseler and Sutter, 1997), a stimulus composed by a matrix of triangles was also used to elicit cortical activity in multifocal VECP studies (Gerth et al., 2003). Triangle patterns were used to reduce the high spatial frequency components present in other forms of stimulation. However, it is difficult to make a straightforward association between the spatial properties of triangle patterns or dartboard patterns and those of sinusoidal gratings that are relevant for the recorded cortical responses. Sinusoidal gratings are the simplest stimuli used to study spatial vision and they were widely used in intracellular and extracellular single-unit electrophysiology, non-invasive electrophysiology, and psychophysics (e.g., Carden et al., 1985; Mullen, 1985; Lee et al., 1989).

Gerth etal. (2003) showed that chromatic pseudo-random VECP had the same polarity nevertheless the presentation mode used to elicit the cortical response, but in their study it lacking the comparison with luminance VECPs. Based in the findings of the effects of the pattern mode presentation on conventional VECPs we could expect that different pseudo random VECP waveforms would be elicited by achromatic and chromatic stimulus for trichromat observers, and color-blind subjects would have decrease or absent responses for chromatic stimulation.

Some studies indicated that the use of non-linear analysis with the separation of the visual response in different states of adaptation (kernels) permit to investigate the presence of mechanisms in the cortical response with different physiological properties distributed in the different kernels (Crewther and Crewther, 2010; Araújo et al., 2013). In the present study, we applied the paradigm of Gerth et al. (2003) by using sinusoidal gratings in order to evaluate how the presentation mode affects luminance and chromatic pseudo-random VECPs. Responses obtained from normal trichromats and red-green congenital color-blinds were compared. A short communication comprising some results of this work was previously presented in the ARVO Annual Meeting (Souza et al., 2012).

\section{MATERIALS AND METHODS SUBJECTS}

All procedures were approved by the Ethic Research Committee of the Tropical Medicine Nucleus, Federal University of Para (Protocol \#023/2011). Eleven normal trichromats $(21.28 \pm 1.86$ years old) and 17 red-green congenital color-blinds (eight protans and nine deutans) were monocularly tested. None of the subjects had previous visual or neurological diseases. We evaluated subject color vision using the Ishihara Plates (1997 38-plate edition; Kanehara \& Co Ltd, Tokyo, Japan), an anomaloscope (HMCAnomaloskop model 47715, Oculus Optikgeräte GmbH, Wetzlar, Germany), and by measuring their color discrimination thresholds (Cambridge Colour Test, Cambridge Research System Ltd, Rochester, England, UK).

\section{STIMULATION}

Visual stimulation, bioelectric recording, and data extraction were performed using a Veris Science 6.10 system (Electro-Diagnostic Imaging, Inc., Redwood City, CA, USA). We presented luminance and compound horizontal gratings, $8^{\circ}$ of visual angle, 2 cycles/degree in a CRT display with $75 \mathrm{~Hz}$ frame rate and $1280 \times 1204$ pixels spatial resolution (FlexScan T662, Eizo, Ishikawa, Japan).

We used sine-wave gratings for the luminance test. A yellow chromaticity (CIE 1976 color space: $u^{\prime}=0.276, v^{\prime}=0.545$ ) was modulated with $99 \%$ Michelson contrast, mean luminance of $10 \mathrm{~cd} / \mathrm{m}^{2}$. The background was a homogeneous field with the same chromaticity and mean luminance.

We used two chromaticities for the test with compound gratings, $r e d\left(u^{\prime}=0.432, v^{\prime}=0.527\right)$ and green $\left(u^{\prime}=0.12, v^{\prime}=0.564\right.$; Figure 1A). For each half cycle of the stimulus the luminance changed sinusoidally from 5 to $10 \mathrm{~cd} / \mathrm{m}^{2}$ and back at the same time that the chromaticity changed sinusoidally from green or red to the intermediate yellow (Figure 1B). The chromatic contrast was modulates along a protan confusion line and at about five away from a deutan confusion line in the CIE 1976 color space. The background had the same stimulus yellow mean chromaticity but the luminance was kept at $10 \mathrm{~cd} / \mathrm{m}^{2}$ throughout the entire stimulus set. For monitor calibration, we used a CS-100A Colorimeter (Minolta, Osaka, Japan). Compound gratings used in this study was similar but not entirely equal to those used previously by Lee et al. (2011) and Li et al. (2014).

A binary $\mathrm{m}$-sequence $\left(2^{14}-1\right.$ elements) controlled stimulus temporal presentation. We used two stimulus presentation modes: pattern-reversal and pattern-onset. For pattern-reversal, each one of two m-steps had base period of $13.3 \mathrm{~ms}$ and we set each of them to show each frame of the grating stimulus with $180^{\circ}$ phase difference. Most of the multifocal VECP studies used this configuration for pattern-reversal mode (e.g., Baseler and Sutter, 1997). For pattern-onset, each m-step had a base period of $26.6 \mathrm{~ms}$ (two frames). The first m-step presented one frame with the grating followed by another frame with the background. The second m-step showed two consecutive frames with the background. This pattern-onset configuration was used before to improve VECP signal-to-noise ratio (Hoffmann et al., 2003). Therefore, we had four stimulus conditions: luminance pattern-reversal, luminance pattern-onset, 


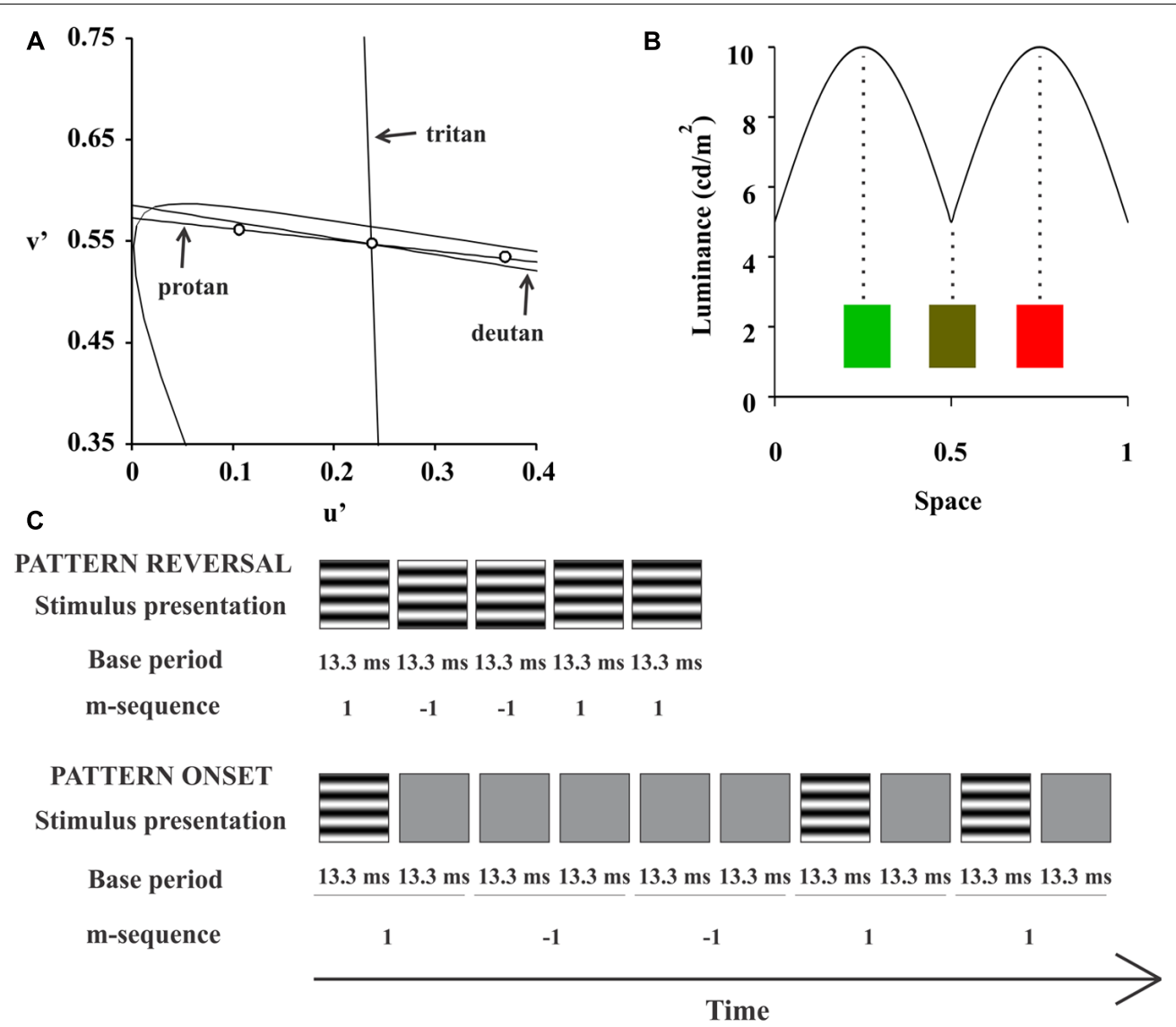

FIGURE 1 | Compound stimuli. For compound stimulation, we used two chromaticities defined in the CIE 1976 Chromaticity Diagram: red $\left(u^{\prime}=0.432, v^{\prime}=0.527\right)$ and green $\left(u^{\prime}=0.12, v^{\prime}=0.564 ;\right.$ (A) For each half cycle of the stimulus, the luminance changed sinusoidally from 5 to
$10 \mathrm{~cd} / \mathrm{m}^{2}$ and back, at the same time that the chromaticity changed sinusoidally from green or red to the intermediate yellow, (B). (C) Shows a space-time diagram of the $\mathrm{m}$-sequence configuration for each presentation mode. red-green pattern-reversal, and red-green pattern-onset. A spacetime diagram of each pattern mode stimulation is shown in the Figure 1C.

\section{RECORDING SETTINGS}

One-channel electroencephalographic signals were recorded using $10 \mathrm{~mm}$ gold surface electrodes (Grass Safelead Gold Disc Electrodes, Grass Technologies, Richmond, USA). Electrode placement followed the standard of the International Society for Clinical Electrophysiology of Vision (ISCEV; Odom et al., 2010): active electrode was placed at $\mathrm{Oz}$, reference electrode at $\mathrm{Fz}$, and ground electrode at Fpz. Continuous recordings were amplified x50,000, on-line filtered between 0.1 and $100 \mathrm{~Hz}$ (P511 Amplifier, Grass Technologies), and digitized at $1.2 \mathrm{kHz}$. For each stimulus condition, we used the Veris Science version 6.010 platform to perform a cross-correlation. The kernel extraction from the VECP was set using the Veris Science software. We extracted the first order kernel (K1), second order kernel first slice (K2.1), and second order kernel second slice (K2.2). The kernel for more information about kernel significance, see Sutter (2000) and Odom (2006). After kernel extraction, the waveforms were low-pass filtered at $50 \mathrm{~Hz}$.

\section{DATA ANALYSIS}

Visual evoked cortical potential kernel waveforms had several positive and negative components starting at about $70 \mathrm{~ms}$ after stimulus onset (Figures 2-4). In order to estimate the evoked response magnitude, we calculated the recording total power. The total power was taken as the numerical integration of the squared amplitude of all amplitude data in the first $500 \mathrm{~ms}$ of the recordings. We used Kruskal-Wallis test with Dunn's post hoc test ( $\alpha=0.05$ ) to compare the magnitude of the VECP components at different stimulus conditions. The $p$-values were corrected by Bonferroni correction.

\section{RESULTS}

\section{VECP WAVEFORMS IN TRICHROMATS}

The VECP waveforms across different kernels varied with stimulus presentation mode and presence of color contrast in the stimulus. Figure 2 shows the VECP kernels waveforms elicited by different stimuli in trichromats. Results obtained from the 11 trichromats were averaged to provide Figure 2 waveforms. For luminance pattern-onset stimulation, $\mathrm{K} 1$ had a negative peak at about $100 \mathrm{~ms}$ followed by a positive peak at about $150 \mathrm{~ms}$. K2.1 and K2.2 also had a negative peak at $100 \mathrm{~ms}$, but the positivity was 


\section{ACHROMATIC}

Pattern Onset

Pattern Reversal

Pattern Onset

Pattern Reversal

K1

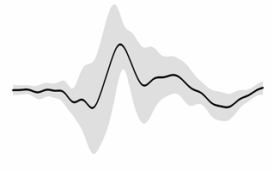

$\mathrm{K}_{2} .1$

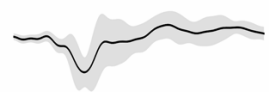

$\mathrm{K} 2.2$

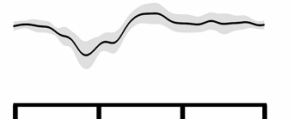

0

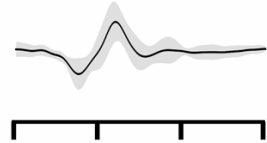

-

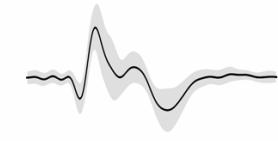

$100 \quad 200 \quad 3000$

Time (ms)
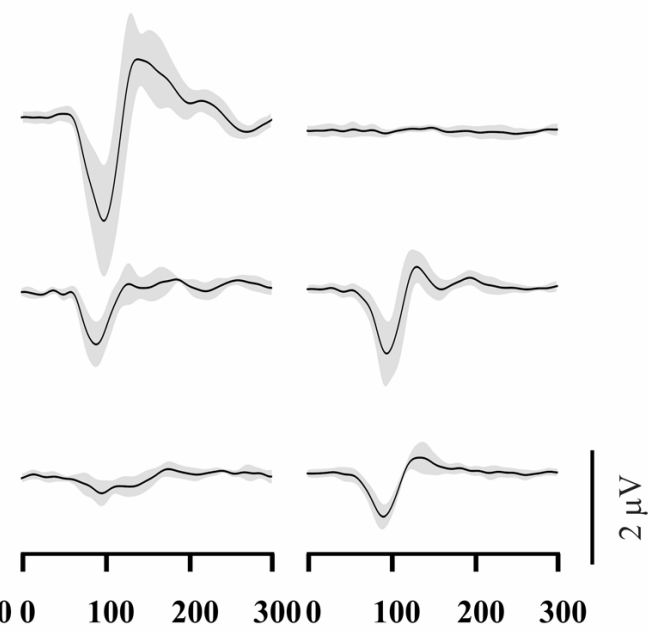

Time (ms)

FIGURE 2 | Visual evoked cortical potential (VECP) average waveforms obtained from normal trichromats using luminance and compound stimuli, as well as pattern-onset and pattern-reversal stimulations. Waveforms were obtained by averaging results from 11 subjects. From top to bottom are depicted waveforms corresponding to the first order kernel (K1), first slice of the second order kernel (K2.1), and second slice of the second

order kernel (K2.2). The average waveforms were dominated by the presence of negativity at about 100 ms for the majority of stimulus conditions and kernels. An important exception is achromatic pattern-reversal K2.1 waveform which is dominated by a positive peak at the same latency. The gray shades represent the SD of the averaged recordings. They provide an indication of interindividual variability.

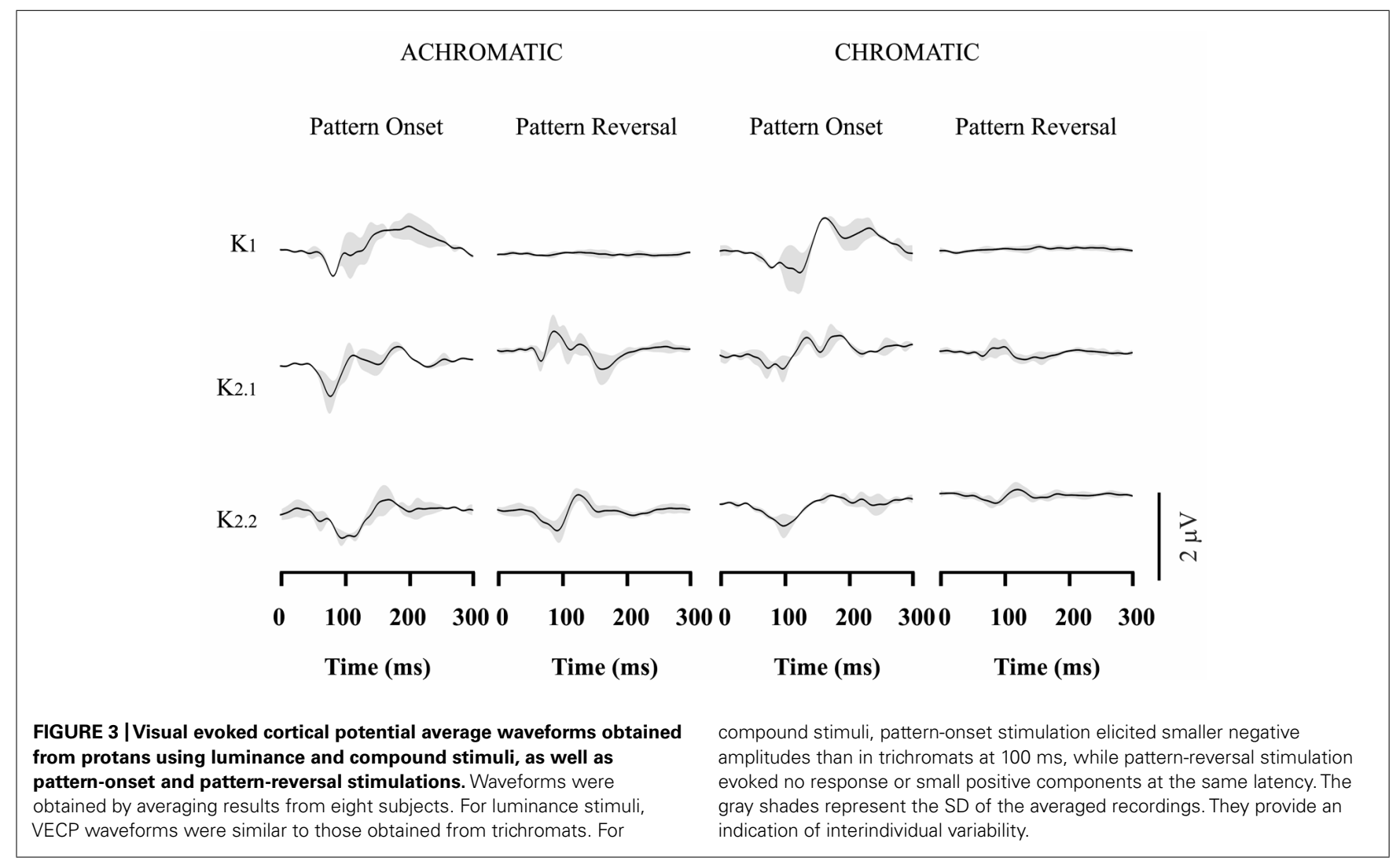




\section{ACHROMATIC}

Pattern Onset

$\mathrm{K} 1$
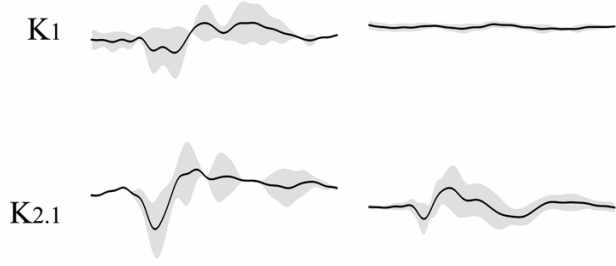

$\mathrm{K} 2.2$

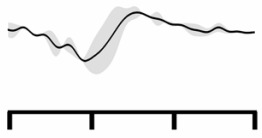

0

100200

Time (ms)

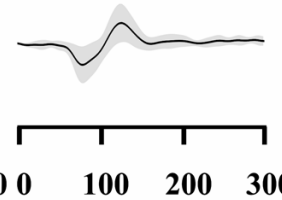

Pattern Reversal
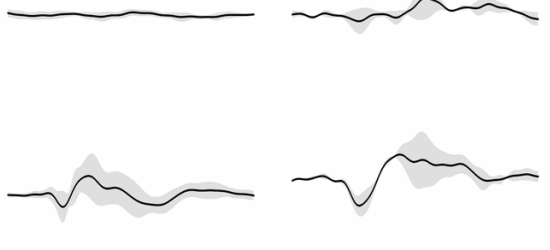

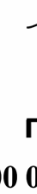

Time (ms)

\section{CHROMATIC}

Pattern Onset Pattern Reversal
FIGURE 4 | Visual evoked cortical potential average waveforms obtained from deutans using luminance and compound stimuli, as well as pattern-onset and pattern-reversal stimulations. Waveforms were obtained by averaging results from 9 subjects. For luminance stimuli, VECP waveforms were similar to those obtained from trichromats. For compound stimuli, pattern-onset stimulation elicited smaller negative amplitudes at $100 \mathrm{~ms}$ than in trichromats, while pattern-reversal stimulation resulted in no response or a small positivity at the same latency. The gray shades represent the SD of the averaged recordings. They provide an indication of interindividual variability. small or entirely missing. For luminance pattern-reversal stimulation, $\mathrm{K} 1$ was very small or absent, K2.1 had a negative peak at about $85 \mathrm{~ms}$ followed by a double-peaked positivity between 100 and $120 \mathrm{~ms}$, and $\mathrm{K} 2.2$ had a negative peak at $100 \mathrm{~ms}$ followed by a positive peak at $130 \mathrm{~ms}$.

For compound pattern-onset stimulation, all kernels waveforms (K1, K2.1, and $\mathrm{K} 2.2$ ) were dominated by negativity at about $100 \mathrm{~ms}$. K1 also had a pronounced positive peak at $150 \mathrm{~ms}$ following the very pronounced main negative component. For compound pattern-reversal, similarly to luminance pattern-reversal stimulation stimulation, $\mathrm{K} 1$ was also very small or absent. In addition, K2.1 and K2.2 were dominated by a negativity occurring at about $100 \mathrm{~ms}$. It should be noted that K2.1 showed opposite polarity for compound pattern-reversal stimulation when compared with luminance pattern-reversal stimulation. It can be interpreted as an indication of differential activation on luminance and chromatic mechanism.

\section{VECP WAVEFORMS IN CONGENITAL RED-GREEN COLOR BLINDS}

Figures 3-4 show VECP waveforms for different kernels obtained by recording from red-green congenital color blinds, either protans (Figure 3) or deutans (Figure 4). As for normal trichromats (Figure 2), VECP was elicited by luminance and compound stimuli as well as by pattern-onset and pattern-reversal stimulation modes. Waveforms were obtained by averaging the results obtained from eight protans and nine deutans, respectively.

Red-green color blinds responses were similar to responses obtained from normal trichromats for both kinds of luminance stimulation. For luminance pattern-onset stimulation, K1 had low-amplitude negative and positive components (slightly larger in protans when compared with deutans), while K2.1 and K2.2 were dominated by a negative peak at about $100 \mathrm{~ms}$. For luminance pattern-reversal stimulation, all kernels were very similar in color blinds and normal trichromats: K1 was very small or absent, K2.1 had a negative peak at about $85 \mathrm{~ms}$ followed by a double-peaked positivity between 100 and $120 \mathrm{~ms}$, and K2.2 had a negative peak at $100 \mathrm{~ms}$ followed by a positive peak at $130 \mathrm{~ms}$.

For compound pattern-onset, K1 was very small or had a small negativity at about $100 \mathrm{~ms}$ in different color-blind subjects, was much smaller in both groups of color blinds when compared with normal trichromats, and was larger in protans when compared with deutans. K2.1 and K2.2 were dominated by a negative peak at $100 \mathrm{~ms}$. K2.1 was small in deutans, slightly larger in protans, and larger in normal trichromats. K2.2 was similar in the three groups.

For compound pattern-reversal, K1 was absent while K2.1 and K2.2 were very small with a small positive component at $100 \mathrm{~ms}$ in both groups of color blinds. Thus, while pattern-reversal K1 was similar in normal trichromats, protans, and deutans, patternreversal K2.1 and K2.2 were very different between color blinds and normal trichromats.

\section{EVALUATION OF THE VECP MAGNITUDE AND ITS COMPARISON AMONG THE KERNELS}

Figures 5-6 show box-plots representing the recording total power in the first $500 \mathrm{~ms}$ elicited by luminance (Figure 5) or compound (Figure 6) stimuli. Results from normal trichromats are 


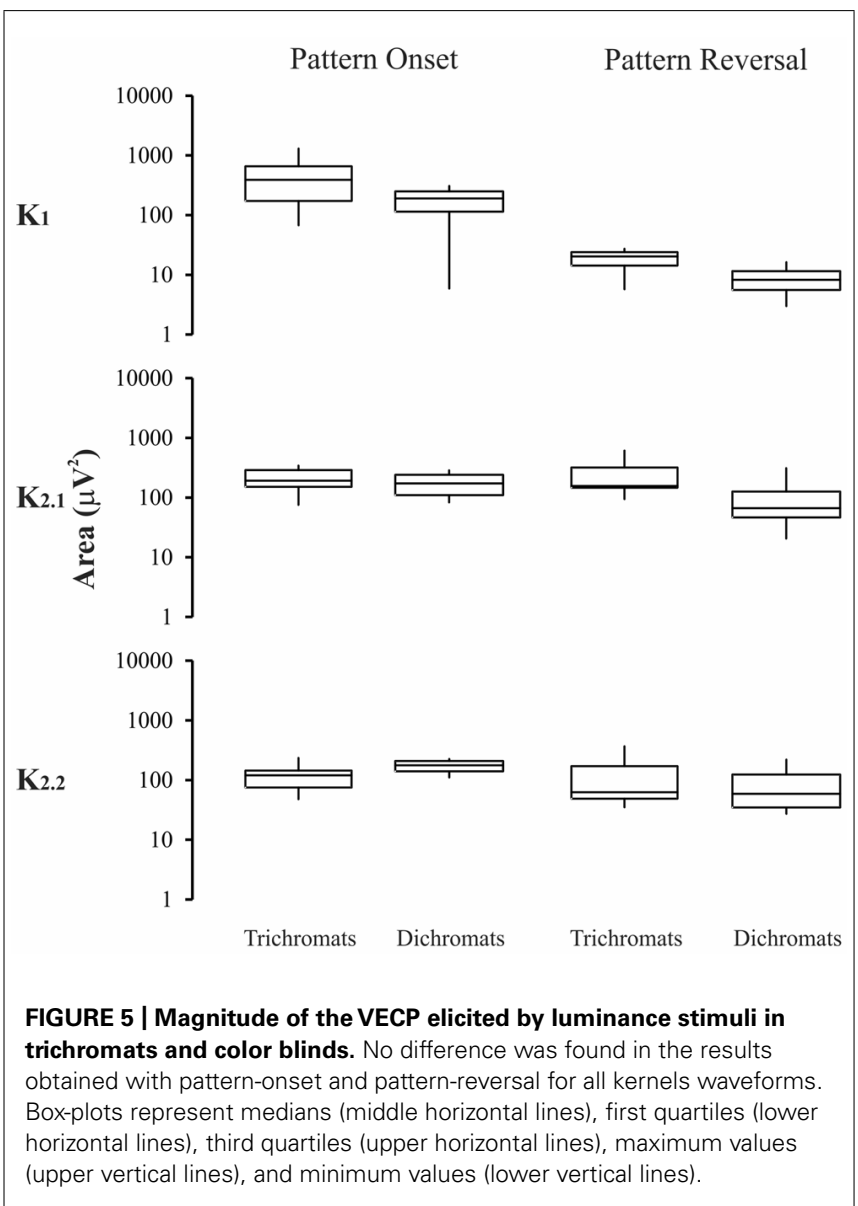

compared with those from red-green color-blinds dichromats. Protans and deutans were grouped together for this comparison with normal trichromats. For luminance stimuli (Figure 5) there were no significant differences $(p>0.05)$ for both patternonset and pattern-reversal stimulation modes and for the three kernels: pattern-onset $\mathrm{K} 1, p=0.7$; pattern-onset $\mathrm{K} 2.1, p=0.16$; pattern-onset $\mathrm{K} 2.2, p=0.35$; pattern-reversal $\mathrm{K} 1, p=0.47$; pattern-reversal K2.1, $p=0.12$; pattern-reversal $\mathrm{K} 2.2$, $p=0.98$.

For compound stimuli (Figure 6) trichromats had significant larger VECP magnitude than color-blinds in the pattern-reversal $\mathrm{K} 2.1$, and pattern-reversal K2.2 $(p<0.05)$. The results of the statistical analysis were as follows: pattern-onset $\mathrm{K} 1, p=0.09$; pattern-onset $\mathrm{K} 2.1, p=0.12$; pattern-onset $\mathrm{K} 2.2, p=0.64$; pattern-reversal $\mathrm{K} 1, p=0.57$; pattern-reversal K2.1, $p=0.002$; pattern-reversal $\mathrm{K} 2.2, p=0.0001)$.

For the dichromats, the kernels of the chromatic waveforms were very similar to those of the achromatic waveforms with slightly reduced amplitude. However, there were no significant differences in amplitudes between the same kernel (or kernel slice).

\section{DISCUSSION}

We used pseudo-random sequences to temporally modulate luminance and compound (luminance plus red-green) sinusoidal gratings presented in pattern-onset and pattern-reversal modes. The present study introduced the use of compound gratings

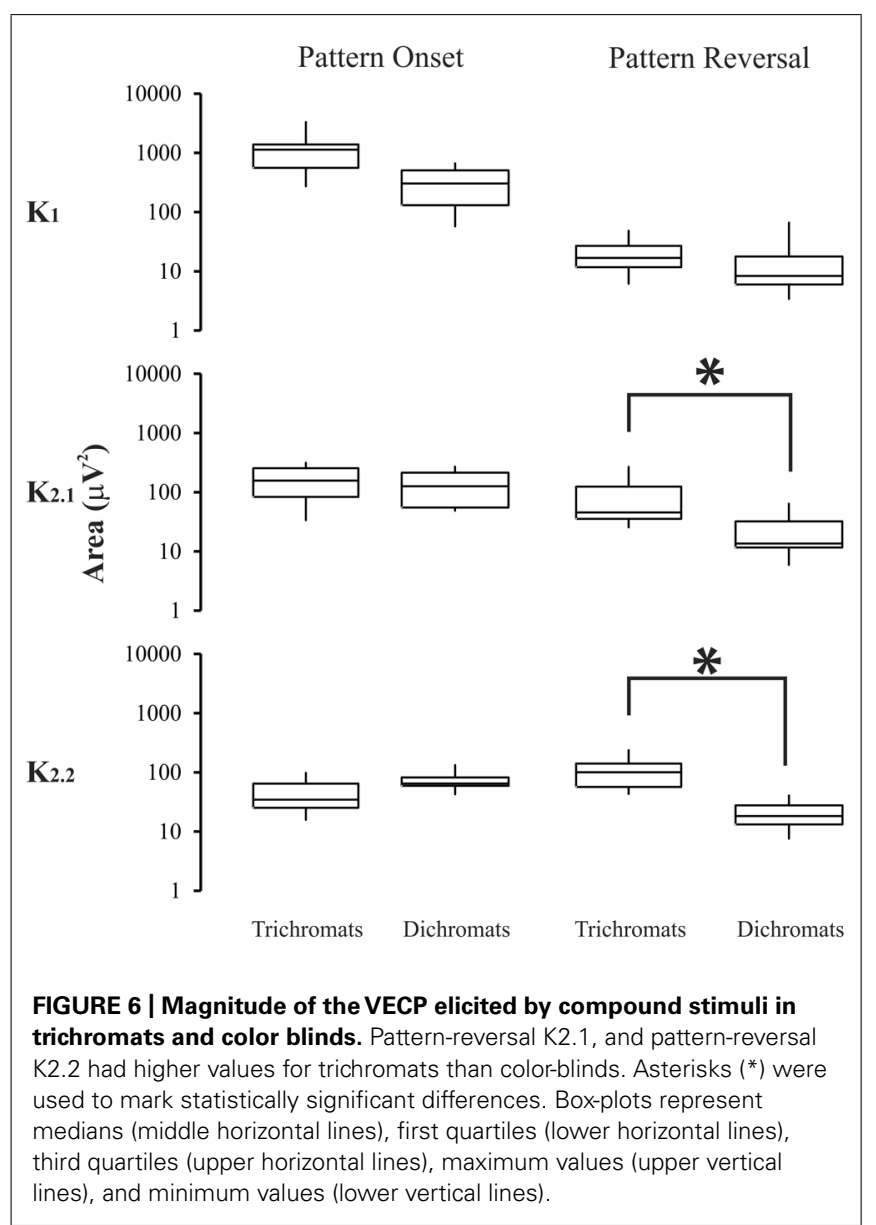

to study the VECP mechanisms. Previously, similar stimuli were used in psychophysical, electroretinographic, and singleunit studies (Lee et al., 2011; Parry et al., 2012; Li et al., 2014). The VECP kernels elicited by pattern-onset and pattern-reversal compound gratings was dominated by negative components at $100 \mathrm{~ms}$. This kind of compound stimuli was previously used in order to simultaneously generate visual responses for luminance and chromatic contrast (Lee et al., 2011). In the present study, we suggest that the responses for compound stimuli were dominated by chromatic information, especially for patternreversal stimulation. Two results support this hypothesis: (i) the pattern-reversal luminance response was dominated by a positive component, while the pattern-reversal compound response was dominated by negativity at the same latency; and (ii) compound stimuli elicited small or no response in red-green congenital color blinds indicating that the cortical response for compound stimuli in the present experiment was dominated by chromatic contribution. Even compound stimuli have been composed by luminance plus chromatic contrast the responses for the present experiments seem to be dominated by chromatic information.

Carden etal. (1985), using conventional VECP, described luminance and chromatic pattern-reversal VECP dominated by positivity at $100 \mathrm{~ms}$ and pattern-onset VECP dominated by negativity for chromatic stimuli and positivity for luminance stimuli at 
the same latency. We have observed that only pattern-reversal stimuli elicited K2.1 with opposite polarity at $100 \mathrm{~ms}$ for luminance or compound contrast, respectively. No difference was observed for pattern-onset in the same kernel at the same latency for these two types of contrast.

Two other previous studies were able to isolate the chromatic response elicited by patterned pseudo-random stimulation (Baseler and Sutter, 1997; Gerth et al., 2003). Baseler and Sutter (1997) observed positive-dominated K2.1 waveforms for patternreversal equiluminant red-green dartboards, while Gerth et al. (2003) described negative-dominated K1 for pattern-onset and negative-dominated K2.1 waveforms for pattern-reversal equiluminant red-green triangle arrays, respectively.

The polarity of pseudo-random luminance VECPs depends on stimulus configuration. K2.1 with positive polarity at $100 \mathrm{~ms}$ was observed with central single hexagon stimulus of high luminance contrast (Klistorner et al., 1997) as well as with high luminance contrast pattern-reversal sinusoidal gratings (Araújo et al., 2013). However, when triangle patterns were used, luminance patternonset $\mathrm{K} 1$ had negative polarity, similarly to chromatic patternonset K1 (Gerth et al., 2003). We found no difference between color blinds and normal trichromats in the amplitude of luminance pattern-onset and pattern-reversal VECP kernels.

Conventional chromatic VECP recorded from congenital redgreen color-blinds were used to evaluate if the response would be dependent of color-opponent mechanisms (Kinney and McKay, 1974; Regan and Spekreijse, 1974; Crognale etal., 1993; Suttle and Harding, 1999; Gomes et al., 2006, 2008). All these studies have found that VECP had small amplitude or was entirely absent for color contrast modulated along color confusion axes. In this work, we observed that the amplitudes at 100 ms of VECP kernels obtained from color blinds by using compound stimuli were much smaller than from normal trichromats. Some color blind subjects exhibited small positivity at $100 \mathrm{~ms}$ evoked by compound patternreversal stimuli, probably reflecting the activation of luminance mechanisms.

Visual evoked cortical potential kernels elicited by patternreversal and pattern-onset stimuli might not represent the same state of adaptation of the visual system (Sutter, 2000). We used base periods of 13.3 and $26.6 \mathrm{~ms}$ for pattern-reversal and pattern-onset stimuli, respectively. The pattern-reversal K2.2 is equivalent to the pattern-onset K2.1, once both of them represent the interaction between two stimulus impulses separated by $26.6 \mathrm{~ms}$. The statistical differences of the VECP amplitude between trichromats and color-blinds for pattern-reversal K2.1, and pattern-reversal K2.2 give support to the suggestion that these kernels are generated by similar chromatic mechanisms.

\section{CONCLUSION}

In conclusion, we suggest that compound pattern-reversal K2.1 is the best stimulus configuration to differentiate between luminance and chromatic mechanisms in VECP studies.

\section{ACKNOWLEDGMENTS}

This research was supported by the following grants: CNPqPRONEX/FAPESPA \#316799/2009; CNPq \#486545/2012-1; and FINEP IBN Net \#1723. Bárbara B. O. Risuenho, Eliza Maria C. B.
Lacerda, Letícia Miquilini received CAPES fellowship for graduate students. Luiz Carlos L. Silveira is a CNPq research fellow.

\section{REFERENCES}

Araújo, C. S., Souza, G. S., Gomes, B. D., and Silveira, L. C. L. (2013). Visual evoked cortical potential (VECP) elicited by sinusoidal gratings controlled by pseudo-random stimulation. PLOS ONE 8:e70207. doi: 10.1371/journal.pone. 0070207

Baseler, H. A., and Sutter, E. E. (1997). M and P components of the VEP and their visual field distribution. Vision Res. 37, 675-690. doi: 10.1016/S00426989(96)00209-X

Berninger, T. A., Arden, G. B., Hogg, C. R., and Frumkes, T. (1989). Separable evoked retinal and cortical potentials from each major visual pathway: preliminary results. Br. J. Ophthalmol. 73, 502-511. doi: 10.1136/bjo.73.7.502

Carden, D., Kulikowski, J. J., Murray, I. J., and Parry, N. R. A. (1985). Human occipital potentials evoked by the onset of equiluminant chromatic gratings. J. Physiol. (Lond.) 369:44P.

Crewther, D. P., and Crewther, S. G. (2010). Different temporal structure for form versus surface cortical color systems-evidence from chromatic non-linear VEP. PLoS ONE 5:e15266. doi: 10.1371/journal.pone.0015266

Crognale, M. A., Switkes, E., Rabin, J., Schneck, M. E., Hægerström-Portnoy, G., and Adams, A. J. (1993). Application of the spatiochromatic visual evoked potential to detection of congenital and acquired color-vision deficiencies. J. Opt. Soc. Am. 10, 1818-1825. doi: 10.1364/JOSAA.10.001818

Dreher, B., Fukada, Y., and Rodieck, R. W. (1976). Identification, classification and anatomical segregation of cells with X-like and Y-like properties in the lateral geniculate nucleus of old-world primates. J. Physiol. 258, 433-452. doi: 10.1113/jphysiol.1976.sp011429

Gerth, C., Delahunt, P. B., Crognale, M. A., and Werner, J. S. (2003). Topography of the chromatic pattern-onset VEP. J. Vision 3, 171-182. doi: 10.1167/3.2.5

Gomes, B. D., Souza, G. S., Lima, M. G., Rodrigues, A. R., Saito, C. A., da Silva Filho, M., et al. (2008). Color discrimination ellipses of trichromats measured with transient and steady-state visual evoked potentials. Vis. Neurosci. 25, 333-339. doi: $10.1017 /$ S0952523808080383

Gomes, B. D., Souza, G. S., Rodrigues, A. R., Saito, C. A., Silveira, L. C. L., and da Silva Filho, M. (2006). Normal and dichromatic color discrimination measured with transient visual evoked potential. Vis. Neurosci. 23, 617-627. doi: $10.1017 /$ S0952523806233194

Gomes, B. D., Souza, G. S., Saito, C. A., da Silva Filho, M., Rodrigues, A. R., Ventura, D. F., et al. (2010). Cone contrast influence on components of the pattern onset/offset VECP. Ophthal. Physiol. Opt. 30, 518-524. doi: $10.1111 / j .1475-1313.2010 .00751 . x$

Gouras, P. (1968). Identification of cone mechanisms in monkey ganglion cells. J. Physiol. 199, 533-547. doi: 10.1113/jphysiol.1968.sp008667

Hicks, T. P., Lee, B. B., and Vidyasagar, T. R. (1983). The responses of cells in macaque lateral geniculate nucleus to sinusoidal gratings. J. Physiol. 337, 183-200. doi: 10.1113/jphysiol.1983.sp014619

Hoffmann, M. B., Straube, S., and Bach, M. (2003). Pattern-onset stimulation boosts central multifocal VEP responses. J. Vision 3, 432-439. doi: 10.1167/ 3.6.4

Kaplan, E., and Shapley, R. M. (1982). X and Y cells in the lateral geniculate nucleus of macaque monkeys. J. Physiol. 330, 125-143. doi: 10.1113/jphysiol.1982. sp014333

Kaplan, E., and Shapley, R. M. (1986). The primate retina contains two types of ganglion cells, with high and low contrast sensitivity. Proc. Natl. Acad. Sci. U.S.A. 83, 2755-2757. doi: 10.1073/pnas.83.8.2755

Kinney, J. A. S., and McKay, C. L. (1974). Test of color-defective vision using the visual evoked response. J. Opt. Soc. Am. 64, 1244-1250. doi: 10.1364/JOSA.64.001244

Klistorner, A., Crewther, D. P., and Crewther, S. G. (1997). Separate magnocellular and parvocellular contributions from temporal analysis of the multifocal VEP. Vision Res. 37, 2161-2169. doi: 10.1016/S0042-6989(97) 00003-5

Kulikowski, J. J., Murray, I. J., and Parry, N. R. A. (1989). Electrophysiological correlates of chromatic-opponent and achromatic stimulation in man. Doc. Ophthalmol. 52, 145-153. doi: 10.1007/978-94-009-2695-0_17

Kulikowski, J. J., and Parry, N. R. A. (1987). Human occipital potentials evoked by achromatic or chromatic checkerboards and gratings. J. Physiol. (Lond.) 388:45P. 
Kulikowski, J. J., Robson, A. G., and McKeefry, D. J. (1996). Specificity and selectivity of chromatic visual evoked potentials. Vision Res. 36, 3397-3401. doi: 10.1016/0042-6989(96)00055-7

Lee, B. B., Martin, P. R., and Valberg, A. (1989). Sensitivity of macaque retinal ganglion cells to chromatic and luminance flicker. J. Physiol. (Lond.) 414, 223-243. doi: 10.1113/jphysiol.1989.sp017685

Lee, B. B., Sun, H., and Valberg, A. (2011). Segregation of chromatic and luminance signals using a novel grating stimulus. J. Physiol. (Lond.) 589, 59-73. doi: 10.1113/jphysiol.2010.188862

Li, X., Chen, Y., Lashgari, R., Bereshpolova, Y., Swadlow, H. A., Lee, B. B., et al. (2014). Mixing of chromatic and luminance retinal signals in primate Area V1. Cereb. Cortex doi: 10.1093/cercor/bhu002 [Epub ahead of print].

McKeefry, D. J., Russell, M. H., Murray, I. J., and Kulikowski, J. J. (1996). Amplitude and phase variations of harmonic components in human achromatic and chromatic visual evoked potentials. Vis. Neurosci. 13, 639-653. doi: $10.1017 /$ S0952523800008543

Mullen, K. T. (1985). The contrast sensitivity of human colour vision to redgreen and blue-yellow chromatic gratings. J. Physiol. (Lond.) 359, 381-400. doi: 10.1113/jphysiol.1985.sp015591

Murray, I. J., Parry, N. R. A., Carden, D., and Kulikowski, J. J. (1987). Human visual evoked potentials to chromatic and achromatic gratings. Clin. Vision Sci. 3, 231-244. doi: 10.1007/BF01184777

Odom, J. V. (2006). "Kernel analysis," in Handbook of Clinical Electrophysiology of Vision Testing, 2nd Edn, eds J. Heckenlively and G. Arden (Cambridge, MA: The MIT Press).

Odom, J. V., Bach, M., Brigell, M., Holder, G. E., McCulloch, D. L., and Tormene, A. P. (2010). ISCEV standard for clinical visual evoked potentials. Doc. Ophthalmol. 120, 111-119. doi: 10.1007/s10633-009-9195-4

Parry, N. R., Murray, I. J., Panorgias, A., McKeefry, D. J., Lee, B. B., and Kremers, J. (2012). Simultaneous chromatic and luminance human electroretinogram responses. J. Physiol. (Lond.) 590, 3141-3154. doi: 10.1113/jphysiol.2011.226951

Porciatti, V., and Sartucci, F. (1999). Normative data for onset VEPs to red-green and blue-yellow chromatic contrast. Clin. Neurophysiol. 110, 772-781. doi: 10.1016/S1388-2457(99)00007-3

Rabin, J., Switkes, E., Crognale, M., Schneck, M. E., and Adams, A. J. (1994). Visual evoked potentials in three-dimensional color space: correlates of spatio-chromatic processing. Vision Res. 34, 2657-2671. doi: 10.1016/0042-6989(94)90222-4
Regan, D., and Spekreijse, H. (1974). Evoked potential indications of colour blindness. Vision Res. 14, 89-95. doi: 10.1016/0042-6989(74)90120-5

Souza, G. S., da Silva, V. G. R., Araújo, C. S., Risuenho, B. B., Gomes, B. D., and Silveira, L. C. L. (2012). "Effects of the presentation mode and color contrast in visual evoked potential elicited by pseudo-random stimuli. Em: annual meeting of the association for research in vision and ophthalmology (ARVO)," in Investigate Ophthalmology Visual Science, Vol. 53 (Rockville: The Association for Research in Vision and Ophthalmology), 5717.

Souza, G. S., Gomes, B. D., Lacerda, E. M. C. B., Saito, C. A., da Silva Filho, M, and Silveira, L. C. L. (2008). Amplitude of the transient visual evoked potential (tVEP) as a function of achromatic and chromatic contrast: contribution of different visual pathways. Vis. Neurosci. 25, 317-325. doi: 10.1017/S09525238 08080243

Sutter, E. E. (2000). The interpretation of multifocal binary kernels. Doc. Ophthalmol. 100, 49-75. doi: 10.1023/A:1002702917233

Suttle, C. M., and Harding, G. F. A. (1999). Morphology of transient VEPs to luminance and chromatic pattern onset and offset. Vision Res. 39, 1577-1584 doi: 10.1016/S0042-6989(98)00223-5

Conflict of Interest Statement: The authors declare that the research was conducted in the absence of any commercial or financial relationships that could be construed as a potential conflict of interest.

Received: 13 August 2014; accepted: 12 January 2015; published online: 28 January 2015.

Citation: Risuenho BBO, Miquilini L, Lacerda EMCB, Silveira LCL and Souza GS (2015) Cortical responses elicited by luminance and compound stimuli modulated by pseudo-random sequences: comparison between normal trichromats and congenital red-green color blinds. Front. Psychol. 6:53. doi: 10.3389/fpsyg.2015.00053

This article was submitted to Perception Science, a section of the journal Frontiers in Psychology.

Copyright (c) 2015 Risuenho, Miquilini, Lacerda, Silveira and Souza. This is an openaccess article distributed under the terms of the Creative Commons Attribution License (CC BY). The use, distribution or reproduction in other forums is permitted, provided the original author(s) or licensor are credited and that the original publication in this journal is cited, in accordance with accepted academic practice. No use, distribution or reproduction is permitted which does not comply with these terms. 